The purpose of this Letters section is to provide rapid dissemination of important new results in the fields regularly covered by Physics of Plasmas. Results of extended research should not be presented as a series of letters in place of comprehensive articles. Letters cannot exceed three printed pages in length, including space allowed for title, figures, tables, references and an abstract limited to about 100 words. There is a three-month time limit, from date of receipt to acceptance, for processing Letter manuscripts. Authors must also submit a brief statement justifying rapid publication in the Letters section.

\title{
A novel phase focusing mechanism in multipactor discharge
}

\author{
R. A. Kishek and Y. Y. Lau \\ Department of Nuclear Engineering and Radiological Sciences, University of Michigan, Ann Arbor, \\ Michigan 48109-2104
}

(Received 31 October 1995; accepted 26 January 1996)

\begin{abstract}
In spite of the mutual repulsion among the space charges, a new phase-focusing mechanism is discovered whereby the leading edge of the multipactor discharge in an rf circuit grows at the expense of the trailing edge. This effect arises from the different impact energies, and hence different secondary electron yields, experienced by different portions of the discharge. This phase focusing mechanism may shape the steady-state multipactor discharge in the form of a very tight bunch of electrons. (C) 1996 American Institute of Physics. [S1070-664X(96)00605-5]
\end{abstract}

Multipactor discharge is a resonant, low to medium voltage phenomenon ${ }^{1-3}$ frequently observed in microwave systems such as rf windows, ${ }^{4}$ accelerator structures, ${ }^{5}$ and rf satellite payloads. ${ }^{6}$ Its persistence requires, at the minimum, stability in the phase of the electron orbit with respect to the rf cycle. ${ }^{1,2}$ Vaughan ${ }^{1}$ established this phase stability for a multipactor electron that is subject to a constant rf electric field. This phase focusing occurs rapidly, over a few rf cycles typically. However, as the space charge builds up, this phase focusing may be destroyed by the mutual electrostatic repulsion among the multipactor electrons. It is conjectured ${ }^{1}$ that the multipactor current may saturate when the electrostatic repulsion is about to overcome the phase focusing that is obtained from the consideration of single-particle orbit.

In this paper, we examine the effect of mutual electrostatic repulsion among the multipactor electrons in an rf circuit. We find that such electrostatic repulsions do not lead to saturation of the multipactor current. More surprisingly, we find that their mutual interactions lead to a new phase focusing mechanism, with the result that the leading multipactor electrons grow at the expense of the trailing ones. This finding is based on a simple model where the multipactor consists of electron sheets moving across a planar gap which is part of the rf circuit. The suppression of the trailing electrons by the leading ones gives credence that, in reality, the steadystate multipactor discharge is in the form of a very tight bunch of electrons, the strength of which is determined primarily by their beam loading ${ }^{3}$ of the rf circuit.

To isolate the physics of mutual repulsion among the multipactor electrons, we consider only two electron sheets, of surface density $\sigma_{1}$ and $\sigma_{2}$, located, respectively, at $x_{1}$ and $x_{2}$ inside a planar gap (Fig. 1). The gap separation is $D$ and the gap voltage is $V_{g}(t)$. We assume that the voltage $V_{g}$ that drives the multipactor is provided by an rf cavity. This cavity, with characteristic frequency $\omega_{0}$ and quality factor $Q$, is modeled by a circuit with circuit elements $R, L$, and $C$. The motions of the multipactor electron sheets induce a wall current, $I_{m}(t)$, which loads this RLC circuit (Fig. 1). Upon impact on a gap surface, the respective incident electron sheet is removed and a new electron sheet is generated by secondary emission.

The evolution of $V_{g}, x_{1}, x_{2}$, and the multipactor current $I_{m}$ is governed by the following normalized equations:

$$
\begin{aligned}
& \left(\frac{d^{2}}{d t^{2}}+\frac{1}{Q} \frac{d}{d t}+1\right) V_{g}(t)=\frac{d}{d t}\left[I_{\mathrm{do}} \sin (\omega t+\phi)+I_{m}(t)\right], \\
& I_{m}(t)=-\sigma_{1} \frac{d x_{1}}{d t}-\sigma_{2} \frac{d x_{2}}{d t}, \\
& \frac{d^{2} x_{1}}{d t^{2}}=V_{g}(t)+\sigma_{1} \cdot\left(x_{1}-\frac{1}{2}\right)+\sigma_{2} \cdot\left[h\left(x_{1}-x_{2}\right)-\left(1-x_{2}\right)\right], \\
& \frac{d^{2} x_{2}}{d t^{2}}=V_{g}(t)+\sigma_{2} \cdot\left(x_{2}-\frac{1}{2}\right)+\sigma_{1} \cdot\left[h\left(x_{2}-x_{1}\right)-\left(1-x_{1}\right)\right],
\end{aligned}
$$

where we used the normalization scales: $D$ for distance, $\omega_{0}$ for frequency, $1 / \omega_{0}$ for time, $v=\omega_{0} D$ for velocity, $U=m v^{2}$ for energy, U/e for voltage, $E=U / e D$ for electric field, and $\Sigma=\epsilon_{0} E$ for surface charge density.

Equation (1) is the circuit equation governing the evolution of the gap voltage, driven by a normalized ideal current source $I_{d}$, (of amplitude $I_{\mathrm{do}}$, frequency $\omega$, and phase $\phi$ at time $t=0$ ) and by the multipactor current $I_{m}$ (Fig. 1). Note that the term $I_{m}$ in Eq. (1) is solely responsible for the nonlinear beam loading and frequency detuning of the cavity by the multipactor. It is simply the wall current induced by the motion of each sheet as given by Eq. (2), where the $\sigma$ 's are always positive by convention. Equation (3a) is the force law for electron sheet 1 . The first term on the right-hand side of (3a) represents the force due to the gap voltage. The second 




FIG. 1. Model of two multipactor electron sheets, interacting among themselves and with the rf circuit.

term, proportional to $\sigma_{1}$, is the force due to sheet 1's image charges on the plates. The final term, proportional to $\sigma_{2}$, represents the force on sheet 1 due to the presence of sheet 2 . It is this term that accounts for the mutual repulsion between the multipactor electrons. In Eq. (3a), $h(s)$ is the unit step function: $h(s)=0, s<0 ; h(s)=1, s>0 ; h(s)=\frac{1}{2}, s=0$. Equation ( $3 b)$ is the force law for electron sheet 2 . It is obtained from Eq. (3a) by interchanging indices 1 and 2 .

On impact with a plate at time $t_{i}$, an incident electron sheet, whether it be sheet 1 or sheet 2 , is removed and a new sheet of surface charge is released by secondary emission. The postimpact surface charge density $\sigma\left(t_{i}^{+}\right)$is related to the pre-impact charge density $\sigma\left(t_{i}^{-}\right)$by

$$
\sigma\left(t_{1}^{+}\right)=\delta \cdot \sigma\left(t_{i}^{-}\right)
$$

where $\delta$ is the coefficient of secondary emission which depends on the electron impact energy, $E_{i}$, of the impacting sheet. Here, $E_{i}=(d x / d t)^{2} / 2$, evaluated at $t=t_{i}^{-}$. For simplicity, we assume that the secondary electrons are emitted with zero velocity. ${ }^{7}$ In addition, we adopt Vaughan's empirical formula ${ }^{8}$ which gives the function plotted in Fig. 2. This shows that $\delta=1$ at two values of impact energies, $E_{1}$ and $E_{2}$, and $\delta>1$ in between. The lower energy $E_{1}$ is designated as the "first crossover point." With a single electron sheet, the steady state obtained by beam loading has been shown ${ }^{3}$ to occur at the first cross-over point (i.e., $\delta=1$ and $E_{i}=E_{1}$ ).

Two mechanisms exist which may overcome the spacecharge forces. The first is the phase-focusing effect of a single particle, described by Vaughan, ${ }^{1}$ and referred to in the first paragraph of this paper. The second, new, mechanism follows. Since each sheet has a different impact energy, it will have a different secondary electron yield, and hence a different growth rate. A simple analysis shows that, kinematically, the leading sheet always arrives at a higher impact energy. For impact energies below $E_{\max },{ }^{9}$ this translates into a higher yield in the leading sheet. Moreover, dynamically, from Fig. 1, we see that the trailing sheet 2 accelerates the

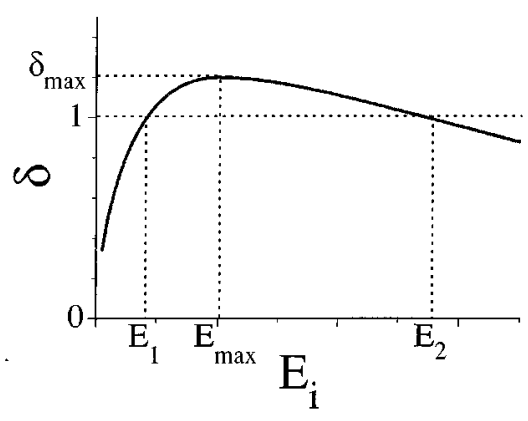

FIG. 2. Secondary electron yield, $\delta$, as a function of impact energy $E_{i}$.

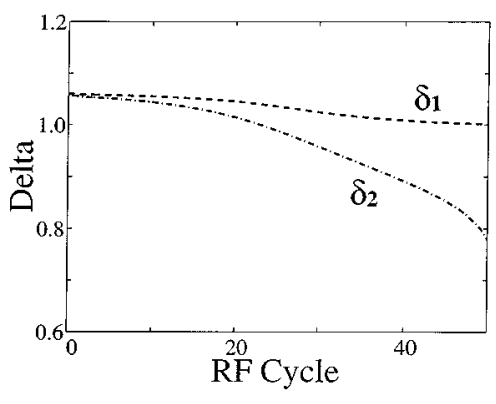

(a)

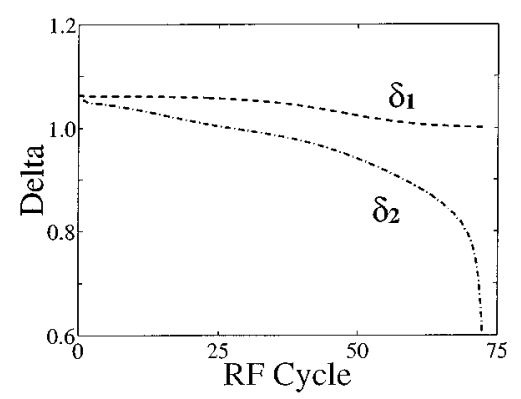

(b)

FIG. 3. Secondary electron yield $\left(\delta_{1}, \delta_{2}\right)$ for sheets 1 and 2 as a function of time: (a) $\sigma_{01}=\sigma_{02}$, (b) $\sigma_{02}=50 \sigma_{01}$.

leading sheet 1 , thus yielding an even higher impact energy and, therefore, a still higher secondary yield, for the leading sheet 1 . On the contrary, the trailing sheet 2 is decelerated by the leading sheet 1 , resulting in a lower impact energy, and therefore, a lower secondary yield for the trailing sheet 2 . Thus growth of the leading sheet is favored and the trailing sheet is, in effect, cannibalized. As we shall see, this effect operates on a longer time scale, typically in tens of rf cycles.

To test this hypothesis we conducted several simulations using Eqs. (1)-(4) and launched sheet 1 slightly ahead of sheet 2 (i.e., sheet 1 is leading) at time $t=0$ (Fig. 1). The gap voltage is selected such that a first-order multipactor can be achieved. In all cases the beam-loading effect dominates ${ }^{3}$ and the steady-state value of the multipactor current is almost the same as that obtained by ignoring the space-charge forces, i.e., by setting both $\sigma_{1}$ and $\sigma_{2}$ equal to zero in Eqs. (3a) and (3b). When the quality factor $Q$ is about 10 or higher, the beam-loading effect saturates the total charge density at such a low level that the space-charge forces never had a chance to overcome Vaughan's phase-focusing mechanism, and the sheets quickly coalesce (within a couple of rf cycles). Therefore the space-charge effect is apparent only in low- $Q$ structures, and we set $Q=1$ henceforth. In Eq. (1), we set $\omega=1$.

Figures 3(a) and 4(a) exhibit the case where both sheets are launched with the same initial charge density, $\sigma_{01}=\sigma_{02}$. Figure 3(a) shows that although both sheets start out with almost the same impact energy and the same secondary electron yield, sheet 2 always has a lower yield. As the charge densities increase, beam loading sets in to reduce the secondary yields of both sheets to near unity (drop in gap voltage leads to lower impact energies). ${ }^{3}$ After some time, the repulsion by sheet 1 forces sheet 2 further and further away from the fixed phase, and the secondary yield for sheet 2 decreases below unity [Fig. 3(a)]. Figure 4(a) shows that $\sigma_{1}$ increases 




(a)

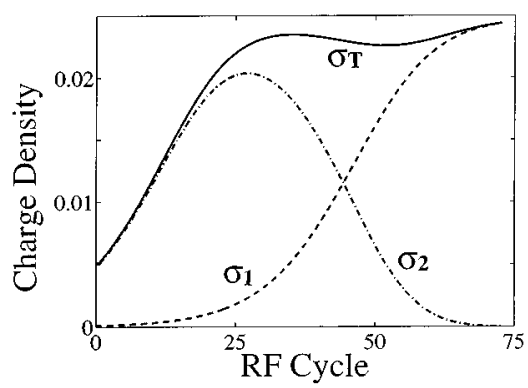

(b)

FIG. 4. Charge density $\left(\sigma_{1}, \sigma_{2}\right)$ on sheets 1 and 2 and the total charge density $\sigma_{T}=\sigma_{1}+\sigma_{2}$ for (a) $\sigma_{01}=\sigma_{02}$, (b) $\sigma_{02}=50 \sigma_{01}$.

rapidly, while $\sigma_{2}$ increases at a slower rate, then drops. Eventually, the charge density $\sigma_{2}$ on sheet 2 becomes so low it can be ignored, and the model reduces to the single sheet model. ${ }^{3}$ By the same token, a model consisting of $N$ electron sheets ( $N$ large) will have its leading sheets growing faster than the trailing sheets, eventually quenching them one by one, until only two sheets, then one, is left. Thus, steady-state multipactor, if it exists, may be adequately described by a single sheet model.

The above cannibalism mechanism is still operative even if the trailing sheet is initially much stronger than the leading sheet. In Figs. 3(b) and 4(b), the trailing sheet 2 is launched with 50 times the initial charge density of the leading sheet 1 . Although the charge density on sheet 2 grows to a relatively high level for some time, sheet 1 dominates in the end. This arises as the yield for sheet 2 is forced to be below unity
[Fig. 3(b)], and $\sigma_{2}$ rapidly diminishes [Fig. 4(b)]. Hence the above process of cannibalism holds, regardless of initial conditions. ${ }^{7}$ These features are also observed for $\delta_{\max }$ (Fig. 2 ) as high as 5. Note, however, that the initial conditions and space-charge forces do affect the timing of the multipactor. A slab of a finite width may reach the steady state more rapidly than a single, infinitesimally thin sheet.

In conclusion, the phase focusing based on the singleparticle theory advanced by Vaughan ${ }^{1}$ and by Riyopoulos et al. ${ }^{2}$ together with the additional phase focusing revealed from the present study, lead to the scenario where steadystate multipactor discharge is in the form of a very tight electron bunch. The level of the steady-state multipactor current in an rf circuit is primarily determined by its loading of the circuit.

\section{ACKNOWLEDGMENTS}

We wish to acknowledge numerous useful discussions with David Chernin, Spilios Riyopoulos, and Ronald Gilgenbach.

This work was supported by the Naval Research Laboratory, Office of Naval Research, and by the Multidisciplinary University Research Initiative, managed by the Air Force Office of Scientific Research and subcontracted through Texas Tech University.

${ }^{1}$ J. R. M. Vaughan, IEEE Trans. Electron Devices ED-35, 1172 (1988).

${ }^{2}$ S. Riyopoulos, D. Chernin, and D. Dialetis, Phys. Plasmas 2, 3194 (1995).

${ }^{3}$ R. Kishek and Y. Y. Lau, Phys. Rev. Lett. 75, 1218 (1995).

${ }^{4}$ A. S. Gilmore, Microwave Tubes (Artech, Norwood, MA, 1986), p. 474.

${ }^{5}$ K. J. Kleman, Proceedings of the Particle Accelerator Conference, Washington, DC, 1993 (Institute of Electronics and Electrical Engineers, New York, 1993), p. 924 (IEEE Catalog No. 93CH32797).

${ }^{6}$ A. D. Woode and J. Petit, Microwave J. January, 142 (1992).

${ }^{7}$ A nonzero initial velocity contributes to a phase perturbation in each cycle, and its inclusion does not lead to a qualitative change of the fixed phase solutions, as was shown in Ref. 2. However, a spread in the emission velocities of the secondary electrons, and the time delays in secondary emission, may weaken (or even destroy in extreme cases) the phasefocusing mechanism.

${ }^{8}$ J. R. M. Vaughan, IEEE Trans. Electron Devices ED-36, 1963 (1989); A. Shih and C. Hor, IEEE Trans. Electron Devices ED-40, 824 (1993).

${ }^{9}$ The analysis in Ref. 3 shows that only the first-crossover point $E_{1}$ of Fig. 2 corresponds to the stable steady-state solution. Hence, we investigate only cases in the vicinity of $E_{1}$. 